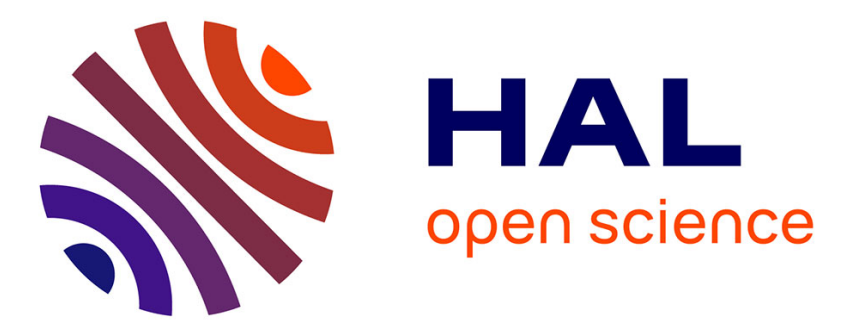

\title{
The Toxoplasma gondii inhibitor-2 regulates protein phosphatase 1 activity through multiple motifs.
}

Quentin Deveuve, Kevin Lesage, Thomas Mouveaux, Mathieu Gissot

\section{To cite this version:}

Quentin Deveuve, Kevin Lesage, Thomas Mouveaux, Mathieu Gissot. The Toxoplasma gondii inhibitor-2 regulates protein phosphatase 1 activity through multiple motifs.. Parasitology Research, 2017, 116 (9), pp.2417-2426. 10.1007/s00436-017-5543-6 . hal-02106427

\section{HAL Id: hal-02106427 https://hal.science/hal-02106427}

Submitted on 23 Apr 2019

HAL is a multi-disciplinary open access archive for the deposit and dissemination of scientific research documents, whether they are published or not. The documents may come from teaching and research institutions in France or abroad, or from public or private research centers.
L'archive ouverte pluridisciplinaire HAL, est destinée au dépôt et à la diffusion de documents scientifiques de niveau recherche, publiés ou non, émanant des établissements d'enseignement et de recherche français ou étrangers, des laboratoires publics ou privés. 


\section{The Toxoplasma gondii inhibitor-2 regulates protein phosphatase 1 activity through multiple motifs.}

\section{Quentin Deveuve ${ }^{1,+}$, Kevin Lesage $^{1,+}$, Thomas Mouveaux ${ }^{1}$ and Mathieu Gissot ${ }^{1,{ }^{*}}$.}

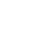

1: Univ. Lille, CNRS, Inserm, CHU Lille, Institut Pasteur de Lille, U1019 - UMR 8204 - CIIL - Centre d'Infection et d'Immunité de Lille, F-59000 Lille, France.

\section{Running Title: T. gondii 12 inhibits PP1 activity}

${ }^{+}$Contributed equally

* Corresponding author: Mathieu GISSOT, Tel: +33 359317430, email: mathieu.gissot@pasteurlille.fr

\section{ACKNOWLEDGEMENTS}

The authors would like to thank Dr. Jamal Khalife for critically reading the manuscript. The authors also thank Dr. Géraldine Tellier for her help setting up the TgPP1 protein purification and the BioImaging Center Lille for access to instruments. This work was supported by Centre National de la Recherche Scientifique (CNRS), Institut National de la Santé et de la Recherche Médicale (INSERM) and a grant from the French National Research Agency (ANR) [grant number ANR-13-JSV3-0006-01 to MG]. The funding bodies had no involvement in the design of the study and collection, analysis, and interpretation of data and in writing the manuscript. 


\section{ABSTRACT}

2 Toxoplasma gondii has a complex life cycle characterized by multiple differentiation steps that

3 are essential for its survival in both human and definitive feline host. Several studies have

4 demonstrated the importance of phosphorylations by protein kinases during the life cycle of $T$.

5 gondii. However, very little is known about protein phosphatases and their regulators in the

6 parasite. We report the molecular and functional characterization of the T. gondii ortholog of

7 the inhibitor-2 protein, designated TgI2. We show that TgI2 encompasses conserved motifs

8 involved in the interaction and modulation of the phosphatase activity of $T$. gondii protein

9 phosphatase 1 , named TgPP1. We show that a specific combination of motifs is involved in binding and/or inhibition of the TgPP1 activity. We show here that the TgI2 protein is a potent inhibitor of TgPP1 phosphatase activity. TgI2 SILK and RVxF motifs are critical for regulating the activity of $\operatorname{TgPP} 1$, a feature that is common with the higher eukaryotes inhibitor- 2 protein

\section{Key words: Toxoplasma; phosphatase; PP1; inhibitor-2.}


2 Toxoplasma gondii is a unicellular eukaryote that infects around a third of the human population

3 worldwide. This parasite belongs to the Apicomplexa phylum which contains other deadly

4 parasite such as Plasmodium (the causative agent of malaria) and Cryptosporidium (responsible

5 for cryptosporidiosis). T. gondii is of clinical importance especially in pregnant women. The

6 first infection can potentially cause severe illness and death in the developing fetus. Moreover,

7 the opportunistic nature of this parasite can lead to encephalitis in immunocompromised

8 individuals and ocular diseases in immunocompetent or immunosuppressed patients (Weiss and

9 Dubey, 2009). T. gondii is acquired primarily through the ingestion of sporulated oocysts containing sporozoites, which are shed by the definitive host (felids) or by ingestion of cystscontaminated meats. In the gastrointestinal tract, the sporozoites or bradyzoites present in the cysts are released and can invade the intestinal epithelium where they differentiate in rapidly dividing tachyzoites. Those tachyzoites disseminate and proliferate during the acute stage of the infection before differentiating in bradyzoites, which encyst in several organs such as muscle tissue or in the central nervous system establishing a chronic infection (Blader et al., 2015). Thus $T$. gondii has a complex life cycle which is characterized by multiple differentiation steps that are essential for its survival in both human and definitive feline host.

Several studies have demonstrated the importance of phosphorylations by protein kinases during the life cycle of $T$. gondii, from invasion (Sugi et al., 2010) to egress (Moudy et al., 2001). In T. gondii tachyzoites, a few protein kinases have been shown to play a role in cell division during endodyogeny (Suvorova et al., 2015)(Berry et al., 2016)(Morlon-Guyot et al., 2014). However, very little is known about protein phosphatases (PPs) and their regulators in the parasite. In other eukaryotes, the protein phosphatase type 1 (PP1), is considered as one of the major phosphatase involved in the control of many dephosphorylation steps. This serine/threonine phosphatase, which exhibits a high degree of conservation throughout 
1 evolution, is known to be involved in numerous essential cellular processes including protein

2 synthesis, actin organization, transcription and cell cycle progression (Ceulemans and Bollen, 2004). It is now known that PP1 localization, activity, and/or specificity are controlled by a large number of interacting-proteins (Peti and Page, 2015). Functional and structural studies revealed that interactions between PP1 and its regulators are mediated by specific binding sites that are conserved throughout evolution, including the so called RVxF and SILK motifs (Wakula et al., 2003)(Huang et al., 1999). So far, about 200 regulators have been described in humans (Hendrickx et al., 2009) while 100 proteins potentially interact with PP1 in Plasmodium (Hollin et al., 2016).

In $T$. gondii, phosphatase inhibitors were used as a proxy to measure the biological role of phosphatases activity. In particular, the use of okadaic acid, an inhibitor of PP2A and PP1, lead to a decreased invasion of the treated tachyzoites suggesting an important role of PP1 in $T$. gondii biology (Delorme et al., 2002). Moreover and similarly to Plasmodium falciparum, only one gene encoding TgPP1 has been identified (Daher et al., 2007). In this study, the authors identified a protein (TgLRR1) homolog to the yeast sds22 protein, known to regulate the yeast PP1 activity (Hong et al., 2000). TgLRR1 belongs to the leucine-rich repeat protein family and interacts with TgPP1 in vitro leading to the inhibition of its activity (Daher et al., 2007).

Other interactors are also conserved and well described in the literature. For example, a small protein called inhibitor-2 (I2) was shown to play an important role in cell cycle regulation of yeast and human (Tung et al., 1995)(Wang et al., 2008). Likewise, an homolog of this protein was identified in P. falciparum (Fréville et al., 2013). This study suggested an essential role for PfI2 for normal growth of the parasite (Fréville et al., 2013). 
1 In the present study, we report the molecular and functional characterization of the T. gondii

2 ortholog of PfI2, designated TgI2. We show that TgI2 encompasses conserved motifs involved

3 in the interaction and modulation of TgPP1 activity.

\section{MATERIALS AND METHODS}

Molecular cloning of TgPPI and TgI2

7 The full length coding region of TgPP1 and TgI2 were amplified by PCR using the primers P1P2 and P3-P4 (Table S1). PCR products were then subcloned in a pJET1.2/blunt vector (Thermofisher) and verified by sequencing for the absence of any modification introduced by Taq polymerase. For the expression of TgPP1 and TgI2, pET28a (Novagen) and pGEX 6p3 (GE Healthcare) expression system were used respectively. Restrictions sites are mentioned in Table S1.

To obtain the TgI2 mutant constructs, we performed a PCR-based site-directed mutagenesis strategy using the pGEX 6p3-TgI2 as template, the primers P5-P6 (TgI2K6A) or P7-P8 (TgI2W37A) or P9-P10 (TgI2R121A/K122A) and Phusion polymerase (NEB). The PCR conditions consisted of $30 \mathrm{~s}$ at $98^{\circ} \mathrm{C}$ followed by 16 cycles at $98^{\circ} \mathrm{C}(5 \mathrm{~s}), 55^{\circ} \mathrm{C}(15 \mathrm{~s})$ and $72^{\circ} \mathrm{C}$ (7min). The parental DNA plasmid was then digested with DpnI. Mutated plasmids were verified by sequencing for the replacement of lysine 6, tryptophan 37, arginine 121 and lysine 122 by an alanine and then used for the expression of mutant TgI2 recombinant proteins.

\section{Recombinant proteins expression}

Protein expression was carried out in the Escherichia coli BL21 strain. For the expression of $\operatorname{TgPP} 1$, culture was induced overnight at $16^{\circ} \mathrm{C}$ in the presence of $0.5 \mathrm{mM}$ isopropyl $\beta$-D-1 thiogalactopyranoside (IPTG) and $1 \mathrm{mM} \mathrm{MnCl}_{2}$. Cells were harvested in sonication buffer 
1 (20mM Tris, 150mM NaCl, 1\% Triton X-100, lysozyme $1 \mathrm{mg} / \mathrm{ml}, 1 \mathrm{mM}$ phenylmethylsulfonyl

2 fluoride (PMSF). 6His-tagged TgPP1 proteins were purified according to manufacturer's instructions by $\mathrm{Ni}^{2+}$ chelation chromatography (Macherey-Nagel). The extract was prepared using a buffer containing $20 \mathrm{mM}$ Tris $\mathrm{HCl}(\mathrm{pH} 7.5), 150 \mathrm{mM} \mathrm{NaCl}, 20 \mathrm{mM}$ Imidazole and $1 \mathrm{mM}$ $\mathrm{MnCl}_{2}$ and loaded on a $1 \mathrm{ml}$ nickel-NTA (Nitrilotriacetic acid) resin column (Macherey Nagel). Elution steps were performed with a buffer containing $20 \mathrm{mM}$ Tris $\mathrm{HCl}(\mathrm{pH} 7.5), 500 \mathrm{mM} \mathrm{NaCl}$, $400 \mathrm{mM}$ Imidazole and $1 \mathrm{mM} \mathrm{MnCl}_{2}$. The imidazole-eluted proteins were dialyzed against the following buffer: $20 \mathrm{mM}$ Tris $\mathrm{HCl}(\mathrm{pH} 7.5), 500 \mathrm{mM} \mathrm{NaCl}$ and $1 \mathrm{mM} \mathrm{MnCl}_{2}$. For the expression of the different forms of $\mathrm{TgI} 2$ (wild-type and mutated), cultures were induced at $37^{\circ} \mathrm{C}$ for $3 \mathrm{~h}$ in the presence of $0.5 \mathrm{mM}$ IPTG. GST-tagged proteins were then purified according to manufacturer's instruction using glutathione-agarose beads (Macherey Nagel). Elution steps were performed with a $10 \mathrm{mM}$ glutathione, $50 \mathrm{mM}$ Tris $\mathrm{HCl}(\mathrm{pH} 8)$ buffer. Under these conditions, the purity checked by sodium dodecyl sulfate polyacrylamide gel electrophoresis (SDS-PAGE) followed by Instant Blue ${ }^{\mathrm{TM}}$ (Expedeon) was $>80 \%$.

Prior to phosphatase activity assays, GST-tagged proteins have been incubated in a buffer containing 50mM Tris $\mathrm{HCl}(\mathrm{pH} 7), 150 \mathrm{mM} \mathrm{NaCl}, 1 \mathrm{mM}$ EDTA and 1mM dithiothreitol (DTT) following the washing steps. After addition of 7 units of PreScission protease (GE Healthcare), the mixture is incubated for $6 \mathrm{~h}$ at $4^{\circ} \mathrm{C}$ allowing the cleavage of the glutathione S-transferase (GST).

\section{GST pull down assays}

Purified TgI2-GST recombinant protein (wild-type or mutated) were bound to glutathioneagarose beads $2 \mathrm{~h}$ at $4^{\circ} \mathrm{C}$ on a rotating wheel and washed with a binding buffer containing $20 \mathrm{mM}$ HEPES (, 20mM Tris $\mathrm{HCl}$ (pH 7.5), 500mM NaCl, 0.2mM EDTA, $1 \mathrm{mM} \mathrm{MnCl}_{2}, 0.1 \%$ Triton $\mathrm{X}-100,1 \mathrm{mM}$ PMSF and $25 \mu \mathrm{g}$ of bovine serum albumin (BSA). Ten $\mu \mathrm{g}$ of TgPP1-6His 
1 recombinant protein were incubated with the TgI2-GST or GST bound to glutathione-agarose

2 beads in binding buffer for $2 \mathrm{~h}$ à $4^{\circ} \mathrm{C}$ on a rotating wheel. After 5 washes with binding buffer,

3 proteins were eluted in SDS-PAGE buffer and loaded on a denaturing 15\% polyacrylamide gel.

4 Separated proteins were then blotted to nitrocellulose. Blots were revealed with anti-His and

5 anti-GST antibodies. Horseradish peroxidase labeled anti-mouse or anti-rabbit

6 immunoglobulins (1:5000) were used as secondary antibodies followed by chemiluminescence

7 detection (Pierce).

8

\section{TgPP1 activity assay}

The activity of recombinant TgPP1 with p-nitrophenyl phosphate (pNPP) as substrate was assayed as previously described (Daher et al., 2007). Initial experiments were performed to determine the optimal conditions for TgPP1 activity. The activity was assessed in a reaction containing 50mM Tris- $\mathrm{HCl}(\mathrm{pH} 7.5)$, 50mM HEPES, $100 \mathrm{mM} \mathrm{NaCl}$, $1 \mathrm{mM}$ DTT, $1 \mathrm{mM} \mathrm{MnCl} 2$ and $0.1 \mathrm{mM}$ Brij35. The reaction was started by adding $5 \mathrm{mM}$ of $\mathrm{pNPP}$. After incubation $1 \mathrm{~h}$ at $37^{\circ} \mathrm{C}$, phosphatase activity was determined spectrophometrically at $405 \mathrm{~nm}$.

To investigate the role of $\mathrm{TgI} 2$ recombinant proteins on TgPP1-6His activity, various concentrations of proteins were added to TgPP1 recombinant protein and preincubated 30min at $37^{\circ} \mathrm{C}$ before testing the TgPP 1 activity. GST was used as control.

\section{Parasite tissue culture and manipulation}

T. gondii strain $\mathrm{RH} \Delta \mathrm{Ku} 80$ tachyzoites were propagated in vitro in human foreskin fibroblasts (HFF) using Dulbeccos's modified Eagles medium supplemented with $10 \%$ fetal calf serum (FCS), $2 \mathrm{mM}$ glutamine, and $1 \%$ penicillin-streptomycin $(100 \mathrm{Units} / \mu \mathrm{L}$ of penicillin and $100 \mu \mathrm{g} / \mu \mathrm{L}$ of streptomycin). T. gondii tachyzoites were grown in ventilated tissue culture flasks at $37^{\circ} \mathrm{C}$ and $5 \% \mathrm{CO}_{2}$. Transgenes were introduced by electroporation into tachyzoites of $T$. 
1 gondii strains and stable transformants were selected by culture in the presence of $2 \mu \mathrm{M}$

2 pyrimethamine. Clonal lines were obtained by limiting dilution.

3 Antibodies

4 The anti-TgENO2 rabbit, anti-TgAlba1 mouse and anti-TgMIC1 mouse antibodies were used 5 at 1:1000 dilution. The anti-HA rabbit antibody (Cell signaling) was used at 1:500 in 6 ImmunoFluorescence assay (IFA) and in Western blots.

Immunofluorescence Assay and confocal imaging

8 Intracellular tachyzoites were fixed with 4\% paraformaldehyde in PBS for 15min, followed by

9 two PBS washes. Parasites were permeabilized with $0.1 \%$ Triton X-100 in PBS containing $0.1 \%$ glycine for $10 \mathrm{~min}$ at room temperature. Samples were blocked with $3 \%$ FCS in the same buffer and the primary antibodies were added on parasites in the same buffer for $1 \mathrm{~h}$ at room temperature. Secondary antibody coupled to Alexa-488 or to Alexa-594 diluted at 1:1000 were added together with DAPI (4',6-diamino-2-phenylindole) for nucleus staining. Confocal imaging was performed with an LSM880 microscope (Zeiss) and a Plan Apochromat objective (Plan-Apochromat 63x:1.40 Oil DIC M27, Zeiss).

\section{Statistical analysis}

The Student t-test was performed to assess the statistical significance of the results. 


\section{Toxoplasma gondii 12 protein encompasses conserved motifs.}

In order to expand our knowledge of TgPP1 regulators repertoire in $T$. gondii, we searched the ToxoDB database (Gajria et al., 2008) for a homolog of the Inhibitor-2 (I2) protein, a well know regulator of PP1 in numerous organisms. We performed a Basic Local Alignment Search Tool (BLAST) analysis (Altschul et al., 1990) using the PfI2 protein sequence and found a protein whose sequence showed $31 \%$ identity on the full length sequence. We aligned the $\operatorname{TgI} 2$ sequence with those of the human and P. falciparum homologs (Fig. 1A) using the MultAlign software (Corpet, 1988). Using the alignment shown in Fig. 1A and the location of known PP1 interacting motifs present on the human and PfI2 proteins we identified three putative PP1 binding motifs in the TgI2 sequence (Fig. 1, boxed). In PfI2, only two motifs were identified and shown to have an impact on its binding to PfPP1 (Fréville et al., 2013), while three functional motifs are found in the human I2 protein (Bollen et al., 2010). By sequence homology and according to the alignment shown in Fig.1A, we found in the TgI2 sequence a first motif, ${ }^{3} \mathrm{VIGK}^{6}$, potentially corresponding to a variation of the sequence of the SILK motif consensus ([SG]ILK). The second motif, ${ }^{33} \mathrm{KHLTW}^{37}$, matched the consensus sequence for the $\mathrm{RVxF}$ motif $([\mathrm{RK}] \mathrm{x}[\mathrm{VI}] \mathrm{x}[\mathrm{FW}])$ with the exception of the valine or isoleucine position. In the human I2, this particular residue is also changed (Fig. 1A) with no impact on the binding properties of this RVxF motif variant (Huang et al., 1999). Finally, we found a third motif $\left({ }^{117} \mathrm{FELKRKQHY}{ }^{125}\right)$ that matches the consensus sequence for the FKK/HYNE motif $(\mathrm{FxxR} / \mathrm{KxR} / \mathrm{KxHY})$.

To better understand the distribution of these PP1 binding motifs among eukaryotic homologs of $\operatorname{TgI} 2$, we gathered the sequence of these proteins in apicomplexan parasites, kinetoplastids, plants and few Opisthokonts and identified their putative PP1 binding motifs (Fig. 1B). 
1 Surprisingly, TgI2 is the only apicomplexan I2 protein to encompass a SILK-like motif, which

2 is found in most Opisthokonts (Human, mouse or Drosophila) but not in plants (Arabidopsis) or Kinetoplastids (Trypanosoma and Leishmania) homologs of the I2 protein. (Fig. 1B).

\section{Toxoplasma gondii 12 protein interacts with TgPP1 through the RVxF motif.}

To test the ability of the TgI2 protein to bind TgPP1, we produced both recombinant proteins with a GST and 6His-tag respectively (Fig. S1A and B). We also produced mutated forms of the TgI2 protein for which each putative motif was mutated (Fig. S1C). To investigate the respective role of each motif, we produced a combination of each mutation, yielding to a total of 7 mutants for the TgI 2 protein. We also checked the purity of each TgI2 mutants and proceeded to a GST-pull down assay (Fig. S1D). In a binding assay, we incubated TgPP1-6His with wild-type or each TgI2-GST mutant coupled to Glutathione-Agarose beads and washed them extensively. We then performed a Western-blot on the eluates of each binding-assay using an anti-6His antibody to identify the TgPP1 protein. We used a Western-blot with an anti-GST antibody to verify the quantity of each TgI2 protein that was originally on the beads. Using this protocol, we showed that the wild-type TgI2 protein is able to bind to TgPP1 (Fig. 2A - lane 3) while the GST alone did not yield detectable TgPP1 signal (Fig. 2A - lane 2). We also showed that the mutation of SILK-like or HYNE motifs of TgI2 protein (Fig. 2A - lanes 4 and 6, respectively) had little change in its TgPP1 binding capacity. In contrast, the mutation of the RVxF motif exhibited a drastic effect, with the TgPP1 signal level close to the background (Fig. 2A - lane 5). The combinatorial mutation of both HYNE and SILK-like resulted in similar results than those obtained with the WT TgI2 (Fig. 2A - lane 8) suggesting that the RVxF motif is the major motif for TgPP1 binding. 
1 While the interaction with TgI2 encompassing any mutation combined with the mutated RVxF

2 resulted in small but close to the background levels of TgPP1 (Fig. 2A - lane 7 and 9), the interaction with the TgI2 protein mutated for the three motifs produced no detectable TgPP1 (Fig. 2A - lane 10). These results suggest that both SILK-like and HYNE motif may contribute to the TgPP1 binding and are therefore secondary binding motifs.

We repeated this experiment three times and measured the intensity of the TgPP1 band for each interaction. We also measured the quantity of $\operatorname{TgI} 2$ for each lane in order to normalize for the quantity of TgI2 protein that was initially on the beads. We therefore calculated a ratio of the intensity of the TgPP1 band over the intensity of the GST-tagged protein that reflects the ability of each GST-tagged protein to bind to TgPP1 (Fig. 2B). These ratio calculated for the mutant lacking the SILK-like and/or HYNE motif indicate that they bind to TgPP1 in a similar manner as the WT TgI2. In contrast, the mutants lacking the RVxF motif show a ten-fold reduction of their TgPP1-binding capacity while the GST and the triple mutant lack the ability to bind to TgPP1 (Fig. 2B). Therefore, these results indicate that the RVxF motif is a major contributor to the TgPP1 binding ability while the HYNE and SILK-like motifs only show a residual contribution.

\section{TgPP1 is an active phosphatase inhibited by okadaic acid.}

To verify if the TgPP1-6His protein we produced was active in our experimental conditions, we performed a colorimetric phosphatase assay using a small phosphorylated peptide. Using the same quantity as starting material, we compared the TgPP1 activity to that of a commercial rabbit PP1 (Fig. 3A). These data showed a robust phosphatase activity increased with concentration of the PP1 protein. TgPP1 activity is undistinguishable from that of the rabbit PP1. When TgPP1 incubation buffer lacked $\mathrm{MnCl}_{2}$, a cation that is indispensable for the PP1 phosphatase activity, we found that the activity measured was independent from the 
1

concentration of TgPP1 added, indicating that TgPP1 activity is dependent on the presence of $\mathrm{MnCl}_{2}$.

To ensure that the phosphatase activity measured in the previous assay was due to a PP1-like phosphatase, we incubated TgPP1 and PfPP1, as a control, in increasing concentration of okadaic acid and measured their phosphatase activity (Fig. 3B) [14]. We found that both TgPP1 and PfPP1 phosphatase activity were inhibited by okadaic acid. We measured an $80 \%$ inhibition of their activity without inhibitor at the highest concentration of okadaic acid. Therefore, the TgPP1 produced in our experimental condition shows a measurable phosphatase activity.

\section{TgI2 acts as an inhibitor of TgPP1 phosphatase activity through its RVxF and SILK} motifs.

To test the regulatory function of $\mathrm{TgI} 2$ on $\mathrm{TgPP} 1$, we performed a phosphatase activity assay in presence of increasing concentration of wild-type TgI2. While the same concentrations of GST alone were not able to induce any significant change in TgPP1 (Fig. S2), increasing concentration of wild-type TgI2 led to a $50 \%$ inhibition of TgPP1 activity (Fig. 4A).

To test the effect of each PP1 binding motif on the inhibitory activity of TgI2, we incubated TgPP1 with increasing concentration of mutated TgI2. First, we tested TgI 2 mutated for the 3 binding motifs and found that this fully mutated version of $\operatorname{TgI} 2$ is unable to significantly alter the TgPP1 activity (Fig. 4B).

In good concordance with the binding-assay, the TgI2 protein mutated for the SILK-like (Fig. 4C) or the HYNE (Fig. 4D) motif alone, kept their ability to inhibit TgPP1 activity. Surprisingly, the single mutation of the RVxF motif was not sufficient to abolish the inhibitory effect of TgI2 on TgPP1 activity (Fig. 4E) although the inhibition level was decreased compare to the wild-type TgI2, suggesting that the SILK-like and/or the HYNE motif are able to convey 
1 the inhibitory function of TgI2. To identify whether both SILK-like and HYNE motifs are necessary for the TgI 2 inhibition of TgPP 1 activity, we used the TgI2 mutants where two out of three motifs are mutated. The TgI2 protein encompassing only the unmutated HYNE motif was unable to inhibit TgPP1 activity, suggesting that this motif alone is not sufficient for the inhibitory function of TgI2 (Fig. 4F). These results indicate that the SILK-like motif alone may be able to mediate the inhibition of the TgPP1 activity. This was confirmed when using a TgI2 mutant for both HYNE and RVxF motifs, which contained the SILK-like motif alone (Fig. 4G). Indeed, this mutant TgI2 protein was able to inhibit the TgPP1 activity (Fig. 4G), indicating its functional role in the TgPP1 activity inhibition.

Finally, we verified whether the TgI2 protein mutated only in the RVxF motif was able to inhibit the TgPP1 activity. In this assay, the TgI2 protein mutated for both HYNE and SILKlike motif was able to significantly inhibit the TgPP1 activity (Fig. 4H), as suggested by the binding-assay.

\section{TgI 2 is located both in the nucleus and the cytoplasm of tachyzoites.}

To gain more information about the function of $\operatorname{TgI} 2$ in the biology of the parasite, we produced an epitope-tagged TgI2-HA strain. We used this strain in IFA and colocalized TgI2-HA with $T$. gondii cellular markers. First, we used a nuclear marker (TgENO2) (Ferguson et al., 2002)and performed an IFA on the TgI2-HA strain. The Fig. 5A shows the colocalization of these proteins and demonstrate that the signal from the HA-tag is localized in the nucleus as well as the cytoplasm. This was further confirmed by the quantitative fluorescence intensity plot that shows that the HA signal is distributed in the nucleus and cytoplasm compartments (Fig. 5B). To confirm this hypothesis, we used a cytoplasmic marker (TgAlba1)(Gissot et al., 2013) in IFA together with an anti-HA antibody. In Fig. 5C, we show a strong codistribution of the signal given by the HA-tag and the cytoplasmic marker. This was further confirmed by the quantitative 
1 fluorescence intensity plot showing that most of the HA signal is localized in the cytoplasm but

2 also in the nucleoplasm (Fig. 5D). To further describe TgI2 localization, we performed a costaining using a microneme marker (TgMIC1). Micronemes are secretory organelles that reside at the apical end of the parasite. We showed that TgI2-HA does not codistribute with micronemes (Fig. S3).

\section{DISCUSSION}

T. gondii genome encodes a gene homolog to the human inhibitor-2 gene. The resulting protein sequence, TgI2, presents motifs that have been found in other I2 proteins to be important for both interaction and regulation of the protein phosphatase 1 catalytic subunit. Surprisingly, while most apicomplexan parasites only present two potential interaction motifs (Fig. 1B, RVxF and HYNE), TgI2 protein sequence may encompass three potential motifs (SILK-like, RVxF and HYNE). The presence of the SILK-like motif is supported by the alignment of the TgI2 and HsI2 protein (Fig. 1A). However the sequence of the SILK-like motif in TgI2 does not correspond to the motif consensus sequence [SG]ILK. The importance of the TgI2 SILKlike motif is revealed by the ability of the TgI2 protein mutated for the two other motifs ( $\mathrm{RVxF}$ and HYNE) to inhibit the TgPP1 activity (Fig. 4G).The distribution of the motif across species may argue for a later acquisition of the SILK domain in Opisthokonts. The presence of the SILK-like domain in the distantly related eukaryote $T$. gondii but not in other Apicomplexan parasites may argue for an independent acquisition and selection of this domain in T. gondii. Other proteins in $T$. gondii display such a structure/function convergence to their mammalian orthologs. For example toxofilin, a protein able to bing G-actin, was shown to exhibit two sequences similar to sequences present in several G- and F-actin-binding proteins, while the third appears to be specific to toxofilin (Jan et al., 2007). Although the SILK-like domain of TgI2 plays a minor role in the interaction with TgPP1, it has an important contribution in the inhibitory activity of this protein (Fig. 4G). The SILK domain was shown to display similar 
1 feature when mutated in the HsI2 protein, with a 500 fold decrease in activity after the Lysine

2 mutation (Park and DePaoli-Roach, 1994; Huang et al., 1999). Similar to what was observed

3 for the HsI2, the SILK-like domain has a little role in the interaction with PP1c but is crucial

4 for its activity (Huang et al., 1999). This apparent discrepancy between binding and inhibition

5 ability through the SILK domain of the I2 proteins may be due to the experimental condition

6 used to assess TgPP 1 activity where binding affinity is not the limiting factor for TgPP1 activity

7 inhibition. The interactions at the SILK domain involve residues 12-17 (KGILK) of the HsI2 protein that bind primarily to a surface created by amino acids 50-59 in the PP1c subunit (Hurley et al., 2007). Structure-function studies have found that the two amino-acids $\left(\mathrm{I}^{14}\right.$ and

$\mathrm{L}^{15}$ ) of the SILK domain are critical for the binding of surface formed by the PP1c $\mathrm{E}^{54}$ and $\mathrm{E}^{56}$ (Hurley et al., 2007). Mutagenesis of either the $\mathrm{I}_{2} \mathrm{I}^{14}$ and $\mathrm{L}^{15}$ or the PP1c $\mathrm{E}^{54}$ and $\mathrm{E}^{56}$ decreased dramatically the PP1c inhibition (Huang et al., 1999; Connor et al., 2000). In the TgI2 protein, the Isoleucine of the SILK domain is conserved while the Leucine is changed to a Glycine. This exchange seems to be neutral for the capacity of the TgI2 SILK-like domain to inhibit the TgPP1 activity, suggesting that there is a flexibility in this amino acid position for the activity of the I2 protein. However, our mutagenesis experiments suggest a crucial involvement of the conserved Lysine $\left(\mathrm{K}^{6}\right)$ in the $T$. gondii ${ }^{3} \mathrm{VIGK}^{6}$ (SILK) motif, since its mutation is sufficient to abolish the SILK-mediated inhibitory activity of TgI2 on TgPP1 (Fig. 4). It has been suggested that the Lysine in the GILK motif $\left(\mathrm{K}^{16}\right)$ of the HsI2 protein is responsible for the proper orientation of the protein when interacting with PP1c therefore enabling the interactions contributed by the $\mathrm{I}^{14}$ and $\mathrm{L}^{15}$ (Hurley et al., 2007). Our study confirms the importance of the terminal Lysine of the SILK-like motif for the activity of TgI2.

The RVxF motif is shared by more than $70 \%$ of the protein binding to PP1c (Hendrickx et al., 2009). However, its consensus sequence [HKR][ACHKMNQRSTV][V][CHKNQRST][FW] defined by Meiselbach et al (Meiselbach et al., 2006).would not include the KSQKW sequence, 
1 a well-established RVxF variant, present in the HsI2 sequence (Hurley et al., 2007). This

2 sequence was nonetheless shown to be crucial for binding and activity of the HsI2 (Huang et al., 1999). The most remarkable change is the replacement of the Valine by a Glutamine which was shown to bind to the PP1 (Hurley et al., 2007) TgI2 ${ }^{33} \mathrm{KHLTW}^{37}$ does match the consensus sequence described earlier with the exception of the Valine that is changed to a Leucine. As for the HsI2 or PfI2 (where the Valine is replaced by an Isoleucine) and based on our mutagenesis and binding assay, this change is not sufficient to prevent the binding of TgI2 to TgPP1. Therefore, the consensus sequence for the $\mathrm{RVxF}$ motif should include other amino-acids at the third position as suggested earlier(Hurley et al., 2007). The RVxF motif of HsI2 was found to bind at least the $\mathrm{D}^{166}$ in HsPP1c within the RVxF binding groove (Hurley et al., 2007). Therefore, the SILK and RVxF motif are predicted to bind different region of the HsPP1c protein. This would explain the complementarity of both SILK and RVxF motifs for the inhibitory activity of $\mathrm{TgI}$. Since the $\mathrm{RVxF}$ groove on HsPP1c is the site of binding of multiple proteins (Egloff et al., 1997), the binding affinity may need to be greater than other site of the I2 protein (e. g. the SILK motif) to accommodate the competition.

The HYNE motif of the HsI2 was found to directly interact with the HsPP1c catalytic domain (Hurley et al., 2007), therefore we anticipated that TgI2 HYNE mutants will show a defect in their inhibitory activity. Moreover, this region of the sequence is highly conserved between HsI2, PfI2 and TgI2. In the assays presented here, the TgI2 protein mutated for the HYNE motif behaved as the wild-type TgI2 protein. This suggests that the HYNE motif has only a minor contribution in the activity of TgI2. Mutagenesis of the HYNE motif on the PfI2 protein resulted in a partial loss of the inhibitory activity of this protein (Fréville et al., 2013), suggesting a smaller contribution than the PfI $2 \mathrm{RVxF}$ motif. However, site directed mutagenesis and deletion of the HYNE motif of the HsI2 protein showed that it was not involved in inhibition of HsPP1c 
1 (Huang et al., 1999). Therefore, depending on the number of PP1 binding site on the protein,

2 the HYNE motif may not be relevant for the activity of I2 homologs.

$3 \mathrm{TgI2}$ was found to localize at both the nuclear and cytoplasmic compartments as shown for the

4 PfI2 protein (Fréville et al., 2013). TgPP1 localization is similarly distributed in both compartments (Daher et al., 2007) which suggests that they may interact in both. We found that the PXTP motif is conserved in the TgI2 protein $\left({ }^{58} \mathrm{PPTP}^{61}\right)$. This motif is known to be phosphorylated during mitosis therefore increasing HsI2 localization to the centrosome (Leach et al., 2003). Phosphopeptides were mapped for the $T$. gondii proteome but no phosphopeptides were recovered for this motif. Instead, two phosphorylated residues were uncovered for TgI2 (S67 and S77) in a region that is poorly conserved. Although, we cannot exclude that phosphorylation of the PXTP motif occurs in T. gondii, there is no evidence for its participation in TgI2 localization.

We show here that the TgI 2 protein is a potent inhibitor of TgPP1 phosphatase activity. TgI2 SILK-like and RVxF motifs are critical for regulating the activity of $\operatorname{TgPP} 1$, a feature that is common with the higher eukaryotes inhibitor-2 protein.

\section{REFERENCES}

Altschul, S. F., Gish, W., Miller, W., Myers, E. W. and Lipman, D. J. (1990). Basic local alignment search tool. J Mol Biol 215, 403-10.

Berry, L., Chen, C.-T., Reininger, L., Carvalho, T. G., EI Hajj, H., Morlon-Guyot, J., Bordat, Y., Lebrun, M., Gubbels, M.-J., Doerig, C. and Daher, W. (2016). The conserved apicomplexan Aurora kinase TgArk3 is involved in endodyogeny, 
duplication rate and parasite virulence. Cellular Microbiology. doi:10.1111/cmi.12571.

Blader, I. J., Coleman, B. I., Chen, C.-T. and Gubbels, M.-J. (2015). Lytic Cycle of Toxoplasma gondii: 15 Years Later. Annual Review of Microbiology 69, 463-485. doi:10.1146/annurev-micro-091014-104100.

Bollen, M., Peti, W., Ragusa, M. J. and Beullens, M. (2010). The extended PP1 toolkit: designed to create specificity. Trends in Biochemical Sciences 35, 450-458. doi:10.1016/j.tibs.2010.03.002.

Ceulemans, H. and Bollen, M. (2004). Functional diversity of protein phosphatase-1, a cellular economizer and reset button. Physiological Reviews 84, 1-39. doi:10.1152/physrev.00013.2003.

Connor, J. H., Frederick, D., Huang, H., Yang, J., Helps, N. R., Cohen, P. T. W., Nairn, A. C., DePaoli-Roach, A., Tatchell, K. and Shenolikar, S. (2000). Cellular Mechanisms Regulating Protein Phosphatase-1: a key functional interaction between inhibitor-2 and type 1 protein phosphatase. Journal of Biological Chemistry 275, 18670-18675. doi:10.1074/jbc.M909312199.

Corpet, F. (1988). Multiple sequence alignment with hierarchical clustering. Nucleic Acids Research 16, 10881-10890.

Daher, W., Oria, G., Fauquenoy, S., Cailliau, K., Browaeys, E., Tomavo, S. and Khalife, J. (2007). A Toxoplasma gondii leucine-rich repeat protein binds phosphatase type 1 protein and negatively regulates its activity. Eukaryotic Cell 6, 1606-1617. doi:10.1128/EC.00260-07. 
Delorme, V., Garcia, A., Cayla, X. and Tardieux, I. (2002). A role for Toxoplasma gondii type $1 \mathrm{ser} / \mathrm{thr}$ protein phosphatase in host cell invasion. Microbes and Infection / Institut Pasteur 4, 271-278.

Egloff, M.-P., Johnson, D. F., Moorhead, G., Cohen, P. T. W., Cohen, P. and Barford, D. (1997). Structural basis for the recognition of regulatory subunits by the catalytic subunit of protein phosphatase 1. The EMBO Journal 16, 1876-1887. doi:10.1093/emboj/16.8.1876.

Ferguson, D. J. P., Parmley, S. F. and Tomavo, S. (2002). Evidence for nuclear localisation of two stage-specific isoenzymes of enolase in Toxoplasma gondii correlates with active parasite replication. International Journal for Parasitology 32, 1399-1410.

Fréville, A., Cailliau-Maggio, K., Pierrot, C., Tellier, G., Kalamou, H., Lafitte, S., Martoriati, A., Pierce, R. J., Bodart, J.-F. and Khalife, J. (2013). Plasmodium falciparum encodes a conserved active inhibitor-2 for Protein Phosphatase type 1: perspectives for novel anti-plasmodial therapy. BMC biology 11, 80 . doi:10.1186/1741-7007-11-80.

Gajria, B., Bahl, A., Brestelli, J., Dommer, J., Fischer, S., Gao, X., Heiges, M., Iodice, J., Kissinger, J. C., Mackey, A. J., Pinney, D. F., Roos, D. S., Stoeckert, C. J., Jr, Wang, H. and Brunk, B. P. (2008). ToxoDB: an integrated Toxoplasma gondii database resource. Nucleic acids research 36, D553-556. doi:10.1093/nar/gkm981.

Gissot, M., Walker, R., Delhaye, S., Alayi, T. D., Huot, L., Hot, D., Callebaut, I., Schaeffer-Reiss, C., Dorsselaer, A. V. and Tomavo, S. (2013). Toxoplasma gondii Alba proteins are involved in translational control of gene expression. Journal of molecular biology 425, 1287-1301. doi:10.1016/j.jmb.2013.01.039. 
Hendrickx, A., Beullens, M., Ceulemans, H., Den Abt, T., Van Eynde, A., Nicolaescu, E., Lesage, B. and Bollen, M. (2009). Docking Motif-Guided Mapping of the Interactome of Protein Phosphatase-1. Chemistry \& Biology 16, 365-371. doi:10.1016/j.chembiol.2009.02.012.

Hollin, T., De Witte, C., Lenne, A., Pierrot, C. and Khalife, J. (2016). Analysis of the interactome of the Ser/Thr Protein Phosphatase type 1 in Plasmodium falciparum. BMC genomics 17, 246. doi:10.1186/s12864-016-2571-z.

Hong, G., Trumbly, R. J., Reimann, E. M. and Schlender, K. K. (2000). Sds22p is a subunit of a stable isolatable form of protein phosphatase 1 (Glc $7 \mathrm{p})$ from Saccharomyces cerevisiae. Archives of Biochemistry and Biophysics 376, 288-298. doi:10.1006/abbi.2000.1715.

Huang, H. -b., Horiuchi, A., Watanabe, T., Shih, S.-R., Tsay, H.-J., Li, H.-C., Greengard, P. and Nairn, A. C. (1999). Characterization of the Inhibition of Protein Phosphatase-1 by DARPP-32 and Inhibitor-2. Journal of Biological Chemistry 274, 7870-7878. doi:10.1074/jbc.274.12.7870.

Hurley, T. D., Yang, J., Zhang, L., Goodwin, K. D., Zou, Q., Cortese, M., Dunker, A. K. and DePaoli-Roach, A. A. (2007). Structural Basis for Regulation of Protein Phosphatase 1 by Inhibitor-2. Journal of Biological Chemistry 282, 28874-28883. doi:10.1074/jbc.M703472200.

Jan, G., Delorme, V., David, V., Revenu, C., Rebollo, A., Cayla, X. and Tardieux, I. (2007). The toxofilin-actin-PP2C complex of Toxoplasma: identification of interacting domains. The Biochemical Journal 401, 711-719. doi:10.1042/BJ20061324. 
Leach, C., Shenolikar, S. and Brautigan, D. L. (2003). Phosphorylation of Phosphatase Inhibitor-2 at Centrosomes during Mitosis. Journal of Biological Chemistry 278, 26015-26020. doi:10.1074/jbc.M300782200.

Meiselbach, H., Sticht, H. and Enz, R. (2006). Structural Analysis of the Protein Phosphatase 1 Docking Motif: Molecular Description of Binding Specificities Identifies Interacting Proteins. Chemistry \& Biology 13, 49-59. doi:10.1016/j.chembiol.2005.10.009.

Morlon-Guyot, J., Berry, L., Chen, C.-T., Gubbels, M.-J., Lebrun, M. and Daher, W. (2014). The Toxoplasma gondii calcium-dependent protein kinase 7 is involved in early steps of parasite division and is crucial for parasite survival: Functional dissection of T. gondii CDPK7 protein. Cellular Microbiology 16, 95-114. doi:10.1111/cmi.12186.

Moudy, R., Manning, T. J. and Beckers, C. J. (2001). The loss of cytoplasmic potassium upon host cell breakdown triggers egress of Toxoplasma gondii. The Journal of Biological Chemistry 276, 41492-41501. doi:10.1074/jbc.M106154200.

Park, I. K. and DePaoli-Roach, A. A. (1994). Domains of phosphatase inhibitor-2 involved in the control of the ATP-Mg-dependent protein phosphatase. The Journal of Biological Chemistry 269, 28919-28928.

Peti, W. and Page, R. (2015). Strategies to make protein serine/threonine (PP1, calcineurin) and tyrosine phosphatases (PTP1B) druggable: Achieving specificity by targeting substrate and regulatory protein interaction sites. Bioorganic \& Medicinal Chemistry 23, 2781-2785. doi:10.1016/j.bmc.2015.02.040. 
Sugi, T., Kato, K., Kobayashi, K., Watanabe, S., Kurokawa, H., Gong, H., Pandey, K., Takemae, H. and Akashi, H. (2010). Use of the kinase inhibitor analog 1NM-PP1 reveals a role for Toxoplasma gondii CDPK1 in the invasion step. Eukaryotic Cell $\mathbf{9}$, 667-670. doi:10.1128/EC.00351-09.

Suvorova, E. S., Francia, M., Striepen, B. and White, M. W. (2015). A novel bipartite centrosome coordinates the apicomplexan cell cycle. PLoS biology 13, e1002093. doi:10.1371/journal.pbio.1002093.

Tung, H. Y., Wang, W. and Chan, C. S. (1995). Regulation of chromosome segregation by Glc8p, a structural homolog of mammalian inhibitor 2 that functions as both an activator and an inhibitor of yeast protein phosphatase 1. Molecular and Cellular Biology 15, 6064-6074.

Wakula, P., Beullens, M., Ceulemans, H., Stalmans, W. and Bollen, M. (2003). Degeneracy and Function of the Ubiquitous RVXF Motif That Mediates Binding to Protein Phosphatase-1. Journal of Biological Chemistry 278, 18817-18823. doi:10.1074/jbc.M300175200.

Wang, W., Stukenberg, P. T. and Brautigan, D. L. (2008). Phosphatase inhibitor-2 balances protein phosphatase 1 and aurora B kinase for chromosome segregation and cytokinesis in human retinal epithelial cells. Molecular Biology of the Cell 19, 48524862. doi:10.1091/mbc.E08-05-0460.

Weiss, L. M. and Dubey, J. P. (2009). Toxoplasmosis: A history of clinical observations. International Journal for Parasitology 39, 895-901. doi:10.1016/j.ijpara.2009.02.004. 
2 Fig. Legends

$3 \quad$ Fig. 1

4 A: Alignment of the T. gondii, P. falciparum and human inhibitor-2 proteins.

5 Sequence alignment of the T. gondii (TGGT1_232760), P. falciparum (Pf3D7_0320000) and 6 human (NP_006232) I2 proteins. Similar amino-acids (aa) are highlighted in gray while 7 identical aa are highlighted in black. The SILK-like (blue), RVxF (red) and HYNE (green) 8 sequence are boxed.

B: Analysis of the distribution of PP1 binding motifs among eukaryotes. Presence $(+)$ or absence (-) of the respective motif is indicated for each species.

Fig. 2: TgPP1 binding assay using different mutant TgI2 proteins.

A: Western blot of the GST-pulled down proteins. The upper panel presents the signal given after hybridization of the anti-His antibody, revealing the TgPP1-6His protein. The middle and bottom panel represent the picture after hybridization of the same membrane using the antiGST antibody, revealing the GST tagged protein (TgI2 and the mutant). On the top of the Fig. is indicated the lane number ( 2 to 10 ) as well as the protein that was used for the GST pulldown. Lane 1 represents the input of the TgPP1 protein before the GST pull-down assay. The three putative TgPP1 binding motifs of TgI2 (SILK-like, RVxF or HYNE) of the WT and each mutant $\operatorname{TgI} 2$ protein are indicated at the top of the Fig.. Molecular weight markers are indicated on the left side of the Fig.. Experiment was repeated three times, a representative experiment is shown. 
B: Quantification of the ratio of the TgPP1-6His signal over the GST tagged protein signal. The signal for the His-tagged protein and GST-tagged protein was quantified using the densitometry tool in the Image Lab software. The ratio of the His-tagged signal over the GST tagged signal is represented here. On the bottom of the Fig. is indicated the protein that was used for the GSTpull down. The three putative TgPP1 binding motifs of TgI2 (SILK-like, RVxF or HYNE) of the WT and each mutant TgI2 protein are indicated at the bottom of the Fig.. A WT motif is indicated in black. A mutated motif is indicated in red and crossed. Experiment was repeated three times. Student's t-test comparing each mutant individually to the WT motifs, *** pvalue: $<<0.001 ;$ mean \pm s.d $(n=3)$.

\section{Fig. 3: TgPP1 is an active phosphatase responsive to okadaic acid inhibition.}

A: Graphical representation of the optic density at $405 \mathrm{~nm}$ measured after an hour of incubation with TgPP1 (blue line) or commercial rabbit PP1 (green line) or TgPP1 without an essential cofactor $\mathrm{MnCl}_{2}$ (yellow line). The x-axis represents the quantity of PP1c used in the assay. Experiment was repeated three times with duplicate measurement.

B: Graphical representation of the TgPP1 (blue line) or PfPP1 (yellow line) activity after incubation with different concentrations of okadaic acid (indicated at the bottom of the Fig.). Activity is represented as a \% of the phosphatase activity measured without inhibitor. Experiment was repeated three times with duplicate measurement.

Fig. 4: TgPP1 activity is inhibited by TgI2 through different motifs.

Graphical representation of the TgPP1 activity after incubation with different concentrations of the TgI2 protein (indicated at the bottom of the Fig.). Activity is represented as a $\%$ of the phosphatase activity measured without inhibitor. The three putative TgPP1 binding motifs of TgI2 (SILK-like, RVxF or HYNE) of the WT and each mutant TgI2 protein are indicated at the top of the Fig.. Experiment was repeated three times with duplicate measurement. Student's t- 
1

2

test, ${ }^{* * *}$ p-value:p $<0.001,{ }^{* *}$ p-value:p $<0.001 ; *$ p-value:p $<0.01 ;$ ns: non-significant; mean \pm s.d $(\mathrm{n}=3)$. A: WT TgI2 protein. B: TgI2 protein mutated at the three sites. C: TgI2 protein mutated for the SILK-like motif. D: TgI2 protein mutated for the HYNE motif. E: TgI2 protein mutated for the RVxF motif. F: TgI2 protein mutated for the SILK-like and RVxF motifs. G: TgI2 protein mutated for the RVxF and HYNE motifs. H: TgI2 protein mutated for the SILK-like and HYNE motifs.

\section{Fig. 5: TgI2 is localized at the cytoplasmic and nuclear compartments.}

A: IFA analysis of the TgI2-HA (green) and TgENO2 (red) localization in the tachyzoite. DAPI (blue) was used to stain DNA. The phase-contrast is also presented. The scale bar is indicated at the bottom right of each picture.

B: Merge of the pictures presented in A with a quantitative fluorescence intensity plot. The Yaxis of the plot represents the fluorescence intensities. The X-axis represents the distance from the beginning to the end of the represented arrow.

C: IFA analysis of the TgI2-HA (green) and TgAlba1 (red) localization in the tachyzoite. DAPI (blue) was used to stain DNA. The phase-contrast is also presented. The scale bar is indicated at the bottom right of each picture.

D: Merge of the pictures presented in $\mathrm{C}$ with a quantitative fluorescence intensity plot. The $\mathrm{Y}$ axis of the plot represents the fluorescence intensities. The X-axis represents the distance from the beginning to the end of the represented arrow. The scale bar is indicated at the bottom right of each picture. 